\title{
An improved model of deformation calculation for circular pressure tunnel with liner in strain-softening rock mass
}

\author{
HAN Jian-xin \\ School of Mathematic and Quantitative Economics \\ Shandong University of Finance and Economics \\ Jinan, China \\ mohan2002@126.com \\ WANG Lei \\ PLA Institute of Engineering Corps \\ Xuzhou, China
}

\begin{abstract}
By considering the interaction of liner and surrounding rock mass, without assuming the support pressure is known a priori, an improved model of solving plastic radius and the distribution of radial displacement around the circular pressured tunnel with liner is proposed. Based on Mohr-Coulomb yield criteria, the results obtained by the strain-softening model are compared with those of elastic perfectly plastic and elastic perfectly brittle models. The examples reveal that the difference of plastic radius obtained by the three models is comparatively smaller, but the differences of radial displacement and hoop stress are comparatively larger, which reveals that the selection of model is very important for predicting distribution of the radial displacement around tunnel. Without assuming the support pressure in advance and being able to consider more factors, the proposed model overcomes some defects of the previous studies and improves the approach of elasto-plastic analysis for tunnel.
\end{abstract}

Keywords----strain-softening model; circular tunnel; liner; deformation calculation; improved model

\section{INTRODUCTION}

In terms of elasto-plastic analysis for circular tunnel based on strain-softening model, Lee Y K et

Foundation item: the National Natural Science Foundation of China (No. 11301298, 51304125, 51474095)

\author{
JIANG Bei \\ School of Civil Engineering and Architecture \\ University of Jinan \\ Jinan China \\ Wang Qi \\ Research Center of Geotechnical and Structural \\ Engineering, \\ Shandong University \\ Jinan China
}

al[1-6] respectively proposed different elastic-plastic analysis methods. But they all assume that support pressure is known a priori, and in practical application, it is needed to measure or assume to obtain the support pressure, which limits the engineering application to a great extent. In addition, they do not consider the interaction between liner and surrounding rock mass and can not consider the influence of internal pressure and the construction time for lining on elasto-plastic solution. So it is necessary and important for elastic-plastic analysis of circular tunnel to build a more reasonable, perfect mechanical model.

In this paper, based on strain softening model, by considering the interaction of the surrounding rock mass and the liner, without assuming support pressure is known a priori, the approach of calculating the plastic radius, radial displacement around circular pressure tunnel with liner is proposed. And the results are compared with those of the elastic-perfectly plastic and elastic-perfectly brittle models.

\section{PROBLEM DESCRIPTION}

As illustrated in Fig.1, a lined circular tunnel is excavated in an infinite rock mass. And the inner and outer radii of the liner are respective $a$ and $b$. The internal pressure imposed on the liner is $p_{0}$ and the support pressure imposed on surrounding rock mass 
is $p_{i}$. If $p_{i}$ is lower than a critical value $p_{i c}$, a plastic zone may be formed around the tunnel. It is notable that $p_{i}$ is unknown in this mechanical model, which is different from previous models[4,5].

$R_{p}$ is assumed to be the plastic radius around the tunnel. $C_{1}, \phi_{1}, E_{1}, v_{1}$ and $G_{1}$ respectively denote the cohesion, frictional angle, elastic modulus, Poisson's ratio and shear modulus of the surrounding rock mass. $C_{2}, \phi_{2}, E_{2}, v_{2}$ and $G_{2}$ respectively denote cohesion, frictional angle, elastic modulus, Poisson's ratio and shear modulus of the liner.

In this work, the following conditions are assumed:

(1) The rock mass is isotropic and a hydrostatic stress field $\sigma_{0}$ is imposed throughout the domain before excavation.

(2)The tunnel is long enough to satisfy the conditions of plane strain.

(3)The radial displacement on the interface of liner and surrounding rock mass is continuous.

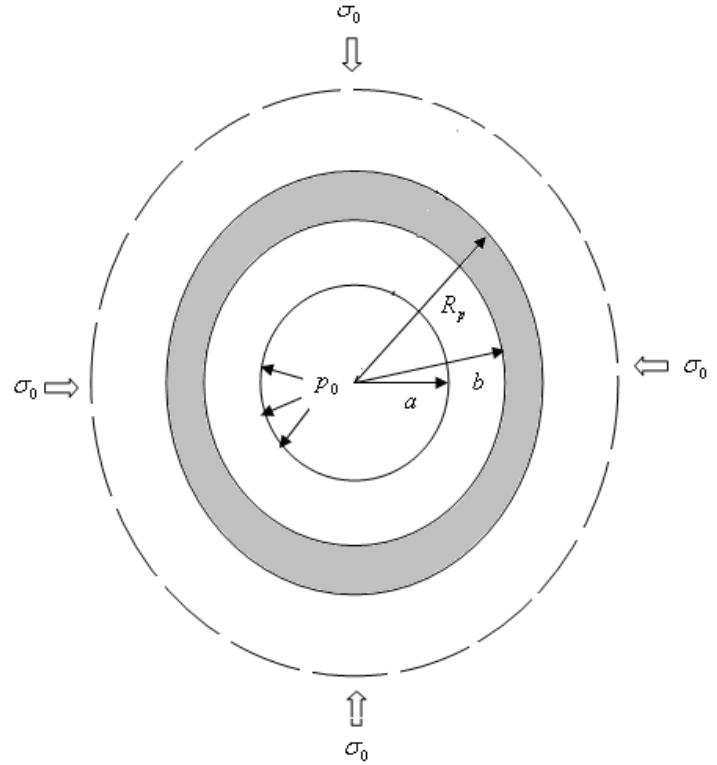

Fig. 1 Mechanical model of circular pressured tunnel with liner

\section{IMPROVEMENT OF ELASTO-PLASTIC ANALYSIS}

One hand, for the surrounding rock mass, for each given support pressure $p_{i}$, using the approach proposed by Lee YK et al[4.5], the corresponding plastic radius, radial displacement and hoop stress of surrounding rock mass can be solved. On the other hand, for the liner, for each $p_{i}$, assuming the solved radial displacement on the interface of liner and surrounding rock mass by Lee YK et al[4.5] is

$$
u=u_{1} \quad(r=b)
$$

If the released radial displacement of inner wall for tunnel before lining is $d$, then the radial displacement of outer wall for liner is $u_{0}=u_{1}-d$. If liner is in elastic state, the following relationship can be concluded:

$$
p_{0}=\frac{p_{i}-m u_{0}}{n}
$$

where

$$
m=\frac{4 G_{2}\left(b^{2}-a^{2}\right)}{b\left[\left(\kappa_{2}-1\right) b^{2}+2 a^{2}\right]}
$$

$n=\frac{\left(\kappa_{2}+1\right) a^{2}}{\left(\kappa_{2}-1\right) b^{2}+2 a^{2}}, \kappa_{2}=3-4 v_{2}$.

Thus, by the connection of intermediate variable $p_{i}$, to every internal pressure $p_{0}$, the corresponding plastic radius and radial displacement of surrounding rock mass can be solved.

\section{EXAMPLES AND DISCUSSION}

The input data of surrounding rock mass are: $E_{1}=10 \mathrm{GPa}, \quad v_{1}=0.25 \quad c_{1}^{p}=1.0 \mathrm{MPa}$, $c_{1}^{r}=0.7 \quad \mathrm{MPa}, \quad \phi_{1}^{p}=30^{0} \quad, \quad \phi_{1}^{r}=22^{0}$ $\varphi_{1}^{p}=\varphi_{1}^{\mathrm{r}}=3.75^{0},^{r}=0.008$. And the input data of liner are: $c_{2}=15.0 \mathrm{MPa}, \phi_{2}=45^{0}, E_{2}=20 \mathrm{GPa}$, $v_{2}=0.25$. The inner and outer radii of liner are respective $a=3 \mathrm{~m}$ and $b=3.3 \mathrm{~m}$. The initial hydrostatic stress $\sigma_{0}=20 \mathrm{MPa}$.

A Plastic radius of surrounding rock mass

Fig.2 shows the relations of plastic radius $R_{p}$ and internal pressure $p_{0}$ for the three models when $p_{0}<6.7309 \mathrm{MPa}$. It can be seen from Fig.2 that the plastic radius is maximum when $p_{0}=0$. With the increase of internal pressure $p_{0}$, the plastic radius gradually decreases. So the existence of internal $p_{0}$ is favorable to the stability of tunnel in the range of $p_{0}<6.7309 \mathrm{MPa}$. The solved plastic radii have some tiny difference among the three different models. The plastic radius solved by elastic-perfectly plastic model is the smallest and that of the elastic-brittle model is the biggest. The plastic radius solved by strain-softening model locates between the other two models. When $p_{0}=0 \mathrm{MPa}$, the dimensionless plastic radii $R_{p} / b$ are respective 1.2915 , 1.2703 and 1.334 for strain-softening, elastic-perfectly plastic and elastic-perfectly brittle 
models. Compared with elastic-perfectly plastic and elastic-perfectly brittle models, the dimensionless plastic radius for strain-softening model respectively increases by $1.67 \%$ and decreases by $3.19 \%$.

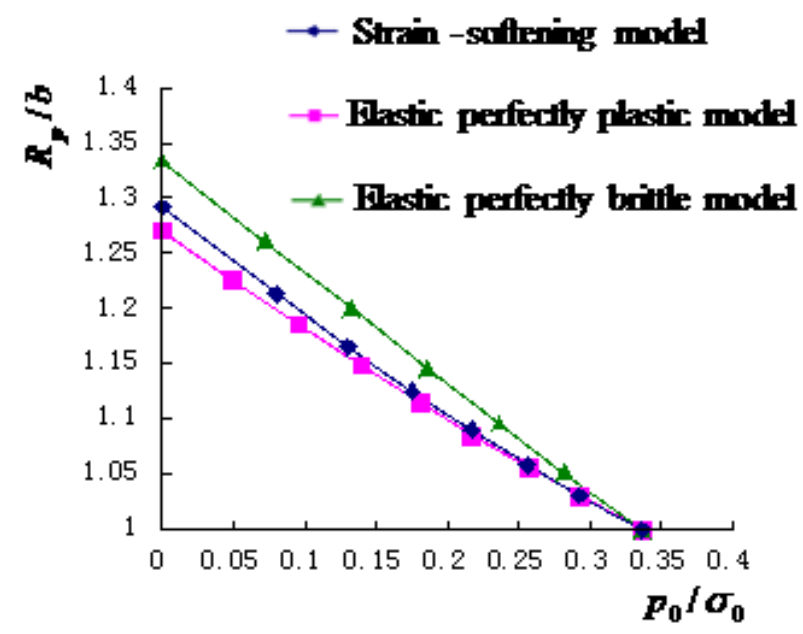

Fig. 2 Relationship of plastic radius and internal pressure

B Radical displacement for surrounding rock mass in plastic zone

Fig.3 shows the ground reaction curves for the three models. It can be seen from Fig.3 that the dimensionless

displacement

$u_{1} / R_{1}\left(2 G_{1}\right) /\left(\sigma_{0}-p_{i c}\right)$ around the tunnel gradually

decreases with the increase of internal pressure $p_{0}$.

When internal pressure $p_{0}$ is the same, the dimensionless displacement solved by elastic-perfectly plastic model is the smallest and that of the elastic-perfectly brittle model is the biggest. The displacement solved by strain-softening model is located between the other two models. When $p_{0}=0$, the dimensionless displacements are respective $1.8669,1.7606$ and 2.0911 for strain-softening, elastic-perfectly plastic and elastic-perfectly brittle models. Compared with elastic-perfectly plastic and elastic-perfectly brittle models, the dimensionless displacement for strain-softening model increases by $6.04 \%$ and decreases by $10.72 \%$, respectively .

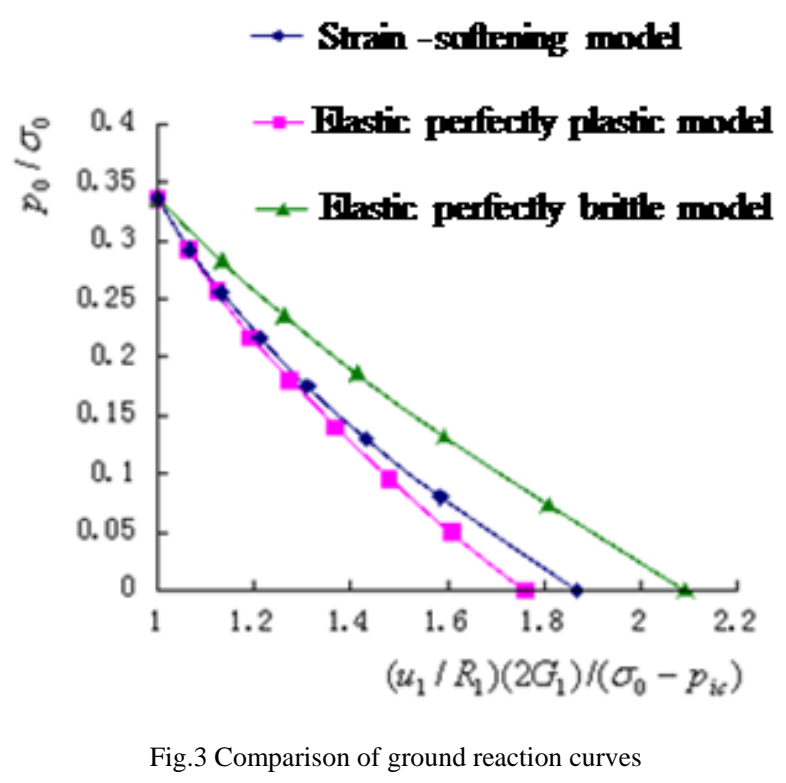

Fig.4 shows the radial displacement $u$ in plastic zone solved by the three models when $p_{0}=2.6000 \mathrm{MPa}$. The radial displacement solved by elastic-perfectly plastic model is the smallest and that of the elastic-perfectly brittle model is the biggest. The radial displacement solved by strain-softening model locates between the other two models. It can be seen from Fig.4 that the radial displacements between strain-softening and elastic-perfectly plastic models have smaller difference, but that between strain-softening and elastic-perfectly brittle models have larger difference. The closer to the cavern, the larger of difference for radial displacements among the three different models is. On the interface of liner and surrounding rock mass, the radial displacements are respective $6.42 \mathrm{~mm}, 6.24 \mathrm{~mm}$ and $7.17 \mathrm{~mm}$ for strain-softening, elastic-perfectly plastic and elastic-perfectly brittle models. Compared with elastic-perfectly plastic and elastic-perfectly brittle models, the radial displacement for strain-softening model increases by $2.88 \%$ and decreases by $10.46 \%$, respectively. 


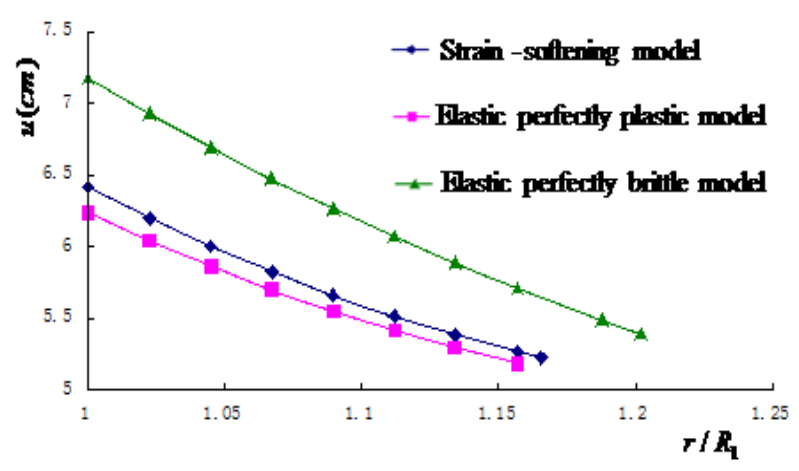

Fig.4 Comparison of displacements in plastic region

\section{CONCLUSIONS}

Based on strain-softening model, without assuming the support pressure is known, by considering the interaction of liner and surrounding rock mass, the approach of solving plastic radius, radial displacement for lined circular tunnel endured internal pressure is proposed. And based on Mohr-Coulomb, the results for strain-softening model are compared with those of elastic-perfectly plastic and elastic-perfectly brittle models. The following conclusions can be drawn:

The plastic radii solved by the three models have tiny difference. The difference increases with the decrease of internal pressure. The plastic radius solved by elastic-perfectly plastic model is the smallest and that of the elastic-perfectly brittle is the biggest. The plastic radius solved by strain-softening model locates between the other two models.

The radial displacements solved by the three models have larger difference. The closer to the cavern, the larger the difference is. The radial displacement solved by elastic-perfectly plastic model is the smallest and that of the elastic-perfectly brittle model is the biggest. The radial displacement solved by strain-softening model locates between the other two models.

\section{REFERENCES}

[1] E. Alonso, L.R. Alejano and F. Varas. Ground response curves for rock masses exhibiting strain-softening behavior.International Journal for Numerical and Analytical Methods in Geomechanics 2003, 27: 1153-1185.

[2] S.L. Wang, X.T. YIN, H. Tang and X.R. Ge. A new approach for analyzing circular tunnel in strain-softening rock masses. International Journal of Rock Mechanics and Mining Sciences
2010,1:170-178.

[3] Q. Zhang, B.S. Jiang and S.L. Wang. Elasto-plastic analysis of a circular opening in strain-softening rock mass. International

$\mathrm{J}$ of Rock Mechanics and Mining Sciences 2012,50:38-46.

[4] Y.K. Lee, and S. Pietruszczak. A new numerical procedure for elasto-plastic analysisof a circular opening excavated in a strain-softening rock mass. Tunnelling Underground Space Technology.2008, 23:588-99.

[5] J.X. Han, S.C. Li, S.C. Li and W.M. Yang. A procedure of strain-softening model for elasto-plastic analysis of a circular opening considering elasto-plastic coupling.Tunnelling

$\begin{array}{lllllllllll}\mathrm{U} & \mathrm{n} & \mathrm{d} & \mathrm{e} & \mathrm{r} & \mathrm{g} & \mathrm{r} & \mathrm{o} & \mathrm{u} & \mathrm{n} & \mathrm{d}\end{array}$ Space Technology., 2013, 37: 128-134.

[6] K.H. Park., B. Tontavanich and J.G. Lee. A simple procedure for ground response curve of circular tunnel in elastic-strain softenng rock masses. Tunnelling Underground Space Technology. 2008, 23: 151-159. 